\title{
New Constraints on all flavour Galactic diffuse neutrino emission with the ANTARES telescope.
}

\section{Timothée Grégoire}

APC, 10 rue Alice Domon et Lenonie Duquet, 75013 Paris , France

E-mail: tgregoireapc.in2p3.fr

\section{Bruny Baret*}

APC, 10 rue Alice Domon et Lenonie Duquet, 75013 Paris , France

E-mail: baretein2p3. fr

\section{on behalf of the ANTARES collaboration.}

\begin{abstract}
The characterization of the high energy neutrino flux from interaction of galactic Cosmic Rays with interstellar matter during their diffusion could shed a new light on Galactic accelerator features, gas distribution morphology and Galactic cosmic ray transport. The central Galactic plane can host an enhanced neutrino production, thus leading to anisotropies in the extraterrestrial neutrino signal as measured by the IceCube Collaboration. The ANTARES neutrino telescope, located in the Mediterranean Sea, offers a favourable view on this part of the sky, thereby allowing for a contribution to the determination of this flux. The expected diffuse Galactic neutrino emission can be obtained linking a model of generation and propagation of cosmic rays with the morphology of the gas distribution in the Milky Way. In this paper, the newly introduced so-called "Gamma model" is assumed as reference. Considering a radially-dependent diffusion coefficient, this model accounts for local cosmic ray measurements, as well as Galactic gamma ray observations. Nine years of ANTARES data are used in this work to search for a possible Galactic contribution to the IceCube neutrino signal. All flavour neutrino interactions are considered, using events with either a track or shower signature. No excess of events is observed and an upper limit is set on the neutrino flux of $1.2-1.1$ times the prediction of the "Gamma model" depending on the cosmic ray spectrum cut-off. This limit excludes the diffuse Galactic neutrino emission as the major cause of the "spectral anomaly" between the two hemispheres measured by IceCube.
\end{abstract}

35th International Cosmic Ray Conference - ICRC2017 -

10-20 July, 2017

Bexco, Busan, Korea

* Speaker. 


\section{Introduction}

The measured diffuse high-energy gamma ray emission along the Galactic plane by the FermiLAT telescope [1] can be attributed above a few $\mathrm{GeV}$ to photons produced in neutral pion decays coming from primary cosmic ray (CR) interactions with the ambient medium (dust, molecular clouds, etc.). A neutrino counterpart emission is also expected from $\pi^{+/-}$decays. The large coverage of the Southern Hemisphere by the ANTARES neutrino telescope, as well as its large effective area and good angular resolution, allows to test this hypothesis. The model "KRA $\gamma$ " or "Gamma model" used in this paper relies on radially-dependant CR diffusion and reproduces local CR features, as well as the diffuse Galactic gamma ray emission measured by Fermi-LAT, H.E.S.S. and Milagro experiments [2,3]. It predicts an expected full sky neutrino flux induced by Galactic CR interactions up to five times higher in the central galactic part than previous models [4]. Consistently with with KASCADE-Grande observations [5], two different energy cut-offs of primary $\mathrm{CR}$ protons, at 5 and $50 \mathrm{PeV}$ respectively, have been considered. They will be referred to as the two "reference models" in this article.

In the last few years, the IceCube Collaboration has reported a significant excess of highenergy neutrinos with respect to the expected atmospheric background $[6,7,8]$. The spectral energy distribution obtained with 4 years of "high-energy starting events" (HESE) through a full sky analysis results in a one flavour normalisation factor $E^{2} \Phi(E)=2.2( \pm 0.7) \cdot(E / 100 \mathrm{TeV})^{-0.58} \times 10^{-8}$ $\mathrm{GeV} \mathrm{cm} \mathrm{s}^{-2} \mathrm{sr}^{-1}$ with a fitted spectral index $\alpha=2.58 \pm 0.25$ [7]. Nevertheless, a dedicated analysis with 6 years of muonic neutrinos from the Northern Hemisphere shows a normalisation factor of $E^{2} \Phi(E)=0.90_{-0.27}^{+0.3} \cdot(E / 100 \mathrm{TeV})^{-0.13} \times 10^{-8} \mathrm{GeV} \mathrm{cm}^{-2} \mathrm{~s}^{-1} \mathrm{sr}^{-1}$ and a spectral index $\alpha=2.13 \pm 0.13$ [9] generating a non-negligible tension between the measured neutrino spectral energy distributions of the two hemispheres, the so-called "spectral anomaly".

As the central region of the Milky Way is at negative declinations, the sum of a Galactic and an extragalactic component $[10,11]$ can result in different spectral behaviours in the two hemispheres.

The ANTARES view of the Southern Sky and its very good angular resolution makes it well suited to either detect the neutrino flux predicted by the reference models or place competitive upper limits. A maximum likelihood analysis is performed assuming the angular and energy distribution of the corresponding signal events and a new stringent upper limit is obtained on the neutrino flux over three decades in energy based on 9 years of data taking.

\section{The ANTARES detector and data sample}

The ANTARES neutrino telescope [12] is installed at $2475 \mathrm{~m}$ depth in the Mediterranean sea, $40 \mathrm{~km}$ off the coast of Toulon, France. Two detection channels are available for neutrinos above a few tens of $\mathrm{GeV}$ : charged current interactions of muonic neutrinos, with the subsequent Cherenkov emission by the outgoing muon, the so-called "track events"; all other interactions, producing electromagnetic or hadronic showers in the detector, lead to so-called "shower events". The former, have a sub-degree angular resolution, an energy reconstruction accuracy of the order of a fraction of a decade and benefit from the kilometer-scale muon track length which enlarges the effective detection. The latter type of events has an angular accuracy of a few degrees and 
an energy resolution of $10 \%$ but these performances are achievable only in a smaller effective detection volume.

The data used in this search have been recorded between the 29th of January 2007 and the 31st of December 2015 for a total livetime of 2423.6 days.

The procedure for suppressing the misreconstructed atmospheric muons and select a sample of Earth through-going neutrino candidates events follows the one used for the search of point-like sources in [13]. The selection of events in this analysis maximizes the detection power of the flux predicted by the reference model with a $50 \mathrm{PeV}$ cut-off. The dataset consists of 7300 tracks and 208 showers

\section{Search Method}

This analysis relies on a likelihood ratio test, similar to the one used in the search for neutrinos from individual point-like or extended sources by ANTARES [14, 15, 16, 17]. It is adapted here to a full-sky search where the signal map is built according to the reference models mentioned above. A probability density function of observables was defined according to given expectations/models. Data are considered to be a mixture of signal and background events, so the likelihood function is defined as:

$$
\mathscr{L}_{\text {sig }+\mathrm{bkg}}=\prod_{\mathscr{T} \in\{\mathrm{tr}, \mathrm{sh}\}} \prod_{i \in \mathscr{T}} \mu_{\mathrm{sig}}^{\mathscr{T}} \cdot p d f_{\mathrm{sig}}^{\mathscr{T}}\left(E_{i}, \alpha_{i}, \delta_{i}\right)+\mu_{\mathrm{bkg}}^{\mathscr{T}} \cdot p d f_{\mathrm{bkg}}^{\mathscr{T}}\left(E_{i}, \theta_{i}, \delta_{i}\right)
$$

where $E_{i}$ is the reconstructed energy, $\alpha_{i}$ and $\delta_{i}$ right ascension and declination (equatorial coordinates), and $\theta_{i}$ zenith angle of the event $i$. For each event topology $\mathscr{T}$ (track or shower), given a total number of events $\mu_{\mathrm{tot}}^{\mathscr{T}}$, the number of background events $\mu_{\mathrm{bkg}}^{\mathscr{T}}$ corresponds to $\mu_{\mathrm{tot}}^{\mathscr{T}}-\mu_{\mathrm{sig}}^{\mathscr{T}}$. The number of signal events $\mu_{\text {sig }}^{\mathscr{T}}$ is fitted when maximising the likelihood, allowing only non-negative values. The signal and background probability density functions of an event are defined as:

$$
\begin{gathered}
p d f_{\mathrm{sig}}^{\mathscr{T}}\left(E_{i}, \alpha_{i}, \delta_{i}\right)=\mathscr{M}_{\mathrm{sig}}^{\mathscr{T}}\left(\alpha_{i}, \delta_{i}\right) \cdot \mathscr{E}_{\mathrm{sig}}^{\mathscr{T}}\left(E_{i}, \alpha_{i}, \delta_{i}\right) \\
p d f_{\mathrm{bkg}}^{\mathscr{T}}\left(E_{i}, \theta_{i}, \delta_{i}\right)=\mathscr{M}_{\mathrm{bkg}}^{\mathscr{T}}\left(\delta_{i}\right) \cdot \mathscr{E}_{\mathrm{bkg}}^{\mathscr{T}}\left(E_{i}, \theta_{i}\right)
\end{gathered}
$$

where $\mathscr{M}^{\mathscr{T}}$ are the probability density functions of an event to be reconstructed in a given position in the sky. The probability density functions $\mathscr{M}_{\text {sig }}^{\mathscr{T}}$, shown in Figure 1 (for the $5 \mathrm{PeV}$ energy cut-off model) obtained from Monte Carlo simulation, depend on the differential neutrino fluxes predicted by the reference models folded with the detector response to a given direction in the sky. The background distribution $\mathscr{M}_{\mathrm{bkg}}^{\mathscr{T}}$ is based on data and only depends on declination since the atmospheric background right ascension distribution is flat because of the Earth's rotation and of the uniform data taking along a sideral day. The probability density function of the reconstructed energy for the signal, $\mathscr{E}_{\text {sig }}^{\mathscr{T}}$ depends on equatorial coordinates as does the energy spectra of the reference models. For the background, $\mathscr{E}_{\mathrm{bkg}}^{\mathscr{T}}$ depends on the corresponding local zenith $\theta_{i}$ to account for potential reconstruction systematic effects due to the detector response.

The test statistic $\mathscr{Q}$ is then defined as the logarithm of the likelihood ratio: 

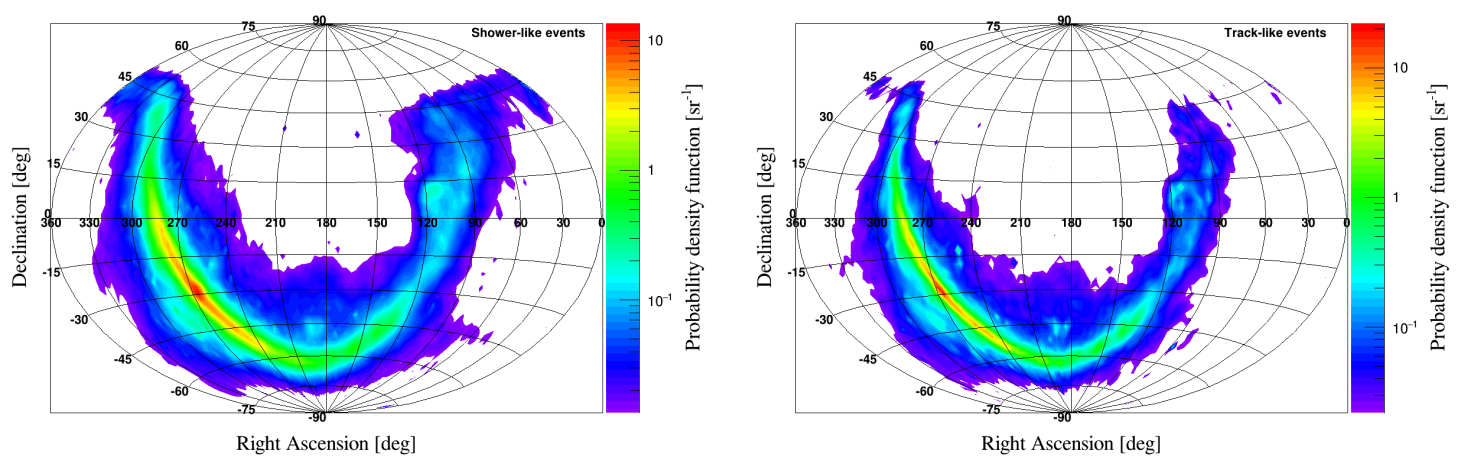

Figure 1: Probability density function of the reconstructed direction of signal events $\mathscr{M}_{\text {sig }}^{\mathscr{T}}$, in equatorial coordinates for shower-like (left) and track-like (right) events.

$$
\mathscr{Q}=\log _{10}\left(\mathscr{L}_{\text {sig+bkg }}\right)-\log _{10}\left(\mathscr{L}_{\text {bkg }}\right)
$$

with $\mathscr{L}_{\text {bkg }}=\mathscr{L}_{\text {sig }+ \text { bkg }}\left(\mu_{\text {sig }}^{\text {sh }}=\mu_{\text {sig }}^{\text {tr }}=0\right)$.

The detection power is computed by building the probability density functions of the test statistic $p d f_{\Phi}(\mathscr{Q})$ for different normalisation factors $\Phi$ of the reference model fluxes. Pseudoexperiments are thus produced, varying the number of signal events $\mu_{\mathrm{sig}}^{\mathrm{sh}+\mathrm{tr}}$ accordingly. They are generated using the probability density functions $\mathscr{M}^{\mathscr{T}}$ and $\mathscr{E}^{\mathscr{T}}$ defined before. A total of $10^{5}$ pseudo-experiments are generated for the background $\left(\mu_{\mathrm{sig}}^{\mathrm{sh}+\mathrm{tr}}=0\right)$ and $10^{4}$ for each value of $\mu_{\mathrm{sig}}^{\mathrm{sh}}$ in the range $[1,55]$ where the rate of showers, taken from the Monte Carlo simulation, is $\sim 20 \%$ of $\mu_{\mathrm{sig}}^{\mathrm{sh}}$.tr. For each pseudo-experiment, the number of fitted track $\left(\mu_{\mathrm{fit}}^{\mathrm{tr}}\right)$ and shower $\left(\mu_{\mathrm{fit}}^{\mathrm{sh}}\right)$ events is recorded. The distribution of $\left[\mu_{\mathrm{sig}}^{\mathrm{sh}+\mathrm{tr}}-\left(\mu_{\mathrm{fit}}^{\mathrm{tr}}+\mu_{\mathrm{fit}}^{\mathrm{sh}}\right)\right]$ has null mean value and a standard deviation $\sigma^{*}=13$ for the model with the $5 \mathrm{PeV}$ cut-off and $\sigma^{*}=12$ with the $50 \mathrm{PeV}$ cut-off. It is worth noticing that the value of $\sigma^{*}$ is related to the background fluctuation, which does not change when varying the true number of signal events for a given model. The probability density functions of $\mathscr{Q}$ for integer numbers of signal events $p d f_{\mu_{\mathrm{sig}}^{\text {sh }+ \text { tr }}}(\mathscr{Q})$ are obtained from pseudo-experiments. They are linked to $p d f_{\Phi}(\mathscr{Q})$, with $\Phi$ leading to a mean number of detected signal events $n$, by:

$$
p d f_{\Phi}(\mathscr{Q})=\sum_{\mu_{\mathrm{sig}}^{\mathrm{sig}+\mathrm{gr}}} P\left(\mu_{\mathrm{sig}}^{\mathrm{sh}+\mathrm{tr}} \mid n\right) \cdot p d f_{\mu_{\mathrm{sig}}^{\mathrm{sh}}}(\mathscr{Q})
$$

where $P$ is the Poissonian probability distribution.

The systematic uncertainty on the acceptance of the ANTARES photomultipliers implies an uncertainty on the effective area of $15 \%$ [21]. To account for this, the number of expected signal events $n$ from a given flux is fluctuated using a Gaussian distribution with a standard deviation of $15 \%$. An uncertainty on the background distribution due to statistical fluctuations in the data is also taken into account by fluctuating $\mathscr{M}_{\mathrm{bkg}}^{\mathscr{T}}\left(\delta_{i}\right)$.

The p-value of a given $\mathscr{Q}$ is defined as the probability to measure a test statistic larger than this one in the background only case. It is given by the anti-cumulative probability density function of $\mathscr{Q}$ with no injected signal (Figure 2). Upper limits at a given confidence level are set according to the corresponding distributions with injected signal events. 


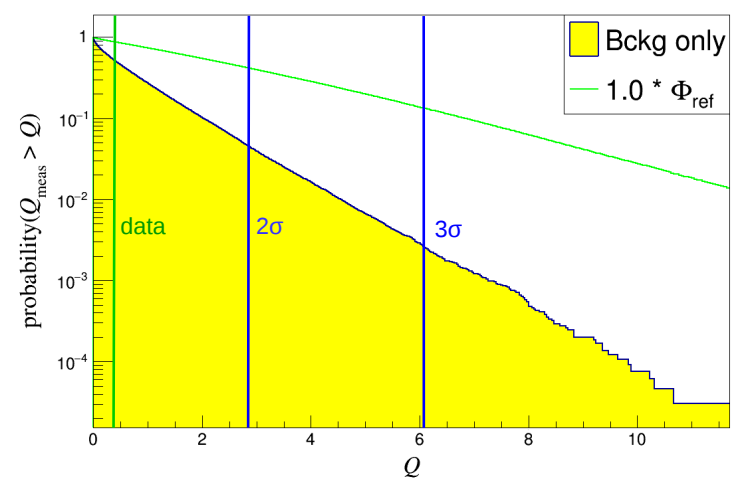

Figure 2: Anti-cumulative distribution of the test statistic $\mathscr{Q}$ from pseudo-experiments for background only (yellow area) and with signal from the reference model with the $5 \mathrm{PeV}$ cut-off (red line). The corresponding values of the test statistic for $2 \sigma$ and $3 \sigma$ confidence level are shown (blue lines) along with the value obtained with data (green line).

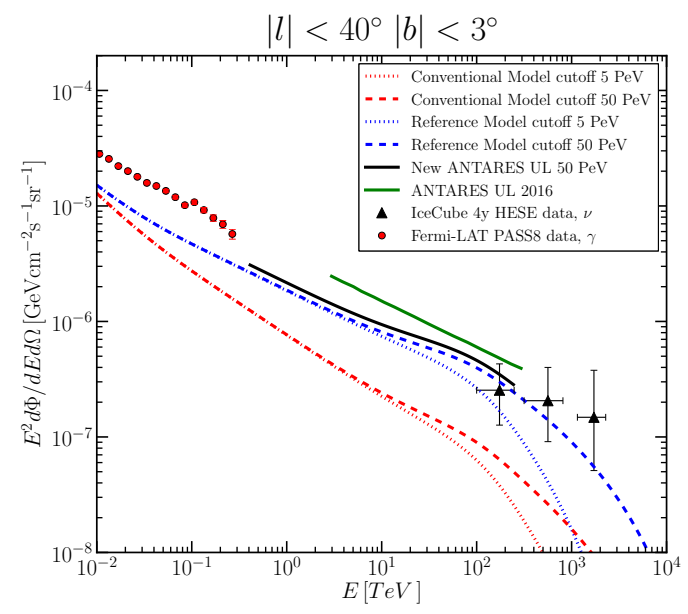

Figure 3: ANTARES upper limit at $90 \%$ confidence level on the three flavour neutrino flux (solid black line) on the reference model with a $50 \mathrm{PeV}$ energy cut-off (blue dashed line). The neutrino fluxes according to the reference model with the $5 \mathrm{PeV}$ energy cut-off (blue dotted line), the conventional model with the $50 \mathrm{PeV}$ (red dashed line) and $5 \mathrm{PeV}$ (red dotted line) cut-offs are shown for all

For the model with the $5 \mathrm{PeV}$ cut-off, $90 \%$ of signal events are in the energy range [0.35,130] $\mathrm{TeV}$ for track-like events and between $[2.0,150] \mathrm{TeV}$ for shower-like events. For the $50 \mathrm{PeV}$ cut-off, these energy ranges are $[0.40,230] \mathrm{TeV}$ for the tracks and $[2.2,260] \mathrm{TeV}$ for the showers. To avoid biasing the analysis, the data have been blinded by time-scrambling. Both the sensitivity and the discovery power of the analysis are derived from this blinded dataset. The sensitivity, defined as the average upper limit at $90 \%$ confidence level, is $1.4 \times \Phi_{\text {ref }}$ when a cut-off for CR primary protons at $5 \mathrm{PeV}$ is set. A mean of $\mu^{*}=11.6$ signal events is expected from the model. It corresponds to the 


\begin{tabular}{|c||c|c||c|c|c|c|}
\hline Energy cut-off & $\mu^{*}$ & $\sigma^{*}$ & $\mu_{\text {data }}^{\text {sh }}$ & $\mu_{\text {data }}^{\text {tr }}$ & p-value & UL at 90\% CL \\
\hline $5 \mathrm{PeV}$ & 11.6 & 13 & 1.9 & $2 \times 10^{-3}$ & 0.67 & $1.1 \times \Phi_{\text {ref }}$ \\
\hline $50 \mathrm{PeV}$ & 13.7 & 12 & 2.6 & $7 \times 10^{-4}$ & 0.54 & $1.2 \times \Phi_{\text {ref }}$ \\
\hline
\end{tabular}

Table 1: Results of the presented analysis for the two reference models corresponding to different energy cut-offs. The number of expected signal events, $\mu^{*}$, is shown, as well as $\sigma^{*}$, the standard deviation of the distribution of the difference between the number of fitted events and the number of injected events in the pseudo-experiment. For the data sample, the numbers of fitted shower-like events, $\mu_{\text {data }}^{\text {sh }}$, and track-like events, $\mu_{\text {data }}^{\text {tr }}$, are reported with the p-values and the upper limits at $90 \%$ confidence level.

sum of track-like and shower-like events, showers constituting $\sim 20 \%$ of the total. The resulting discovery power at $3 \sigma$ confidence level is $7 \%$. For the model with a $50 \mathrm{PeV}$ cut-off, the sensitivity is $1.05 \times \Phi_{\text {ref }}$ and $\mu^{*}=13.7$ signal events are expected, resulting in a discovery power of $14 \%$ for a $3 \sigma$ confidence level.

\section{Results}

After unblinding, the test statistic of the data is computed. The corresponding $\mathscr{Q}$ value is shown as the green line in Figure 2. Table 1 presents the results for the two different cut-off energies (column 1) considered by the models. Column 2 reports the number of expected events, $\mu^{*}$, and column 3 the standard deviation of the distribution of the number of fitted events, $\sigma^{*}$, which are defined in section 3 .

For the data sample, the numbers of fitted track-like events, $\mu_{\text {data }}^{\mathrm{tr}}$, and shower-like events, $\mu_{\mathrm{data}}^{\mathrm{sh}}$, are reported in columns 4 and 5 , respectively. Their sum is smaller than $\mu^{*}$, but still compatible with the expected fluctuations. These include the Gaussian fluctuation due to the background (which is within $1 \sigma^{*}$ ) and the Poissonian fluctuation on the number of signal events.

The p-value of the data - as defined in section 3 - is reported in column 6. The derived upper limits at $90 \%$ confidence level on the reference models are reported in the last column of Table 1.

Figure 3 shows the $90 \%$ confidence level upper limit of this analysis that relies on the particular morphology and energy spectrum of the reference model. The blue line refers to the reference model assuming a cut-off of $5 \mathrm{PeV}$ for the primary protons producing neutrinos when interacting with gas. Although full sky data were used in this analysis, the expectations and the results concerning the inner Galactic plane region $\left(|l|<40^{\circ}\right.$ and $\left.|b|<3^{\circ}\right)$ are shown on this plot. This allows to compare the improvement of the presented limit with respect to the previous ANTARES constraint on the neutrino emission [18] from the same region. The diffuse gamma-ray spectral energy distribution derived from PASS8 Fermi-LAT data [19] obtained after the subtraction of point-like components comprised in this region is also shown for comparison. In the same way, the red dashed line shows the predicted spectrum from the conventional model with homogeneous CR diffusion. The neutrino flux from individual events with origin compatible with this region coming from the 4 year IceCube HESE catalog is shown as the black triangles. Only all flavour neutrino fluxes are 
represented in this figure.

\section{Conclusions}

The study reported here applies a likelihood ratio test on nine years of ANTARES data collected from 2007 to 2015 to search for a diffuse Galactic-dominated neutrino flux, characterised by the recently introduced "Gamma model" used as reference model. As a result, a neutrino flux with normalisation factor of $1.1 \times \Phi_{\text {ref }}$ (resp. $1.2 \times \Phi_{\text {ref }}$ ) is excluded at $90 \%$ confidence level when the model with the $5 \mathrm{PeV}$ cut-off (resp. $50 \mathrm{PeV}$ ) is considered.

Using neutrinos of all flavours as well as a larger amount of data leads to an improvement in the sensitivity and more stringent upper limits with respect to the previous ANTARES analysis [18]. The new upper limits do not extend above $\sim 200 \mathrm{TeV}$ due to the significant softening of the spectrum. The additional gain in sensitivity below $3 \mathrm{TeV}$ with respect to the previous analysis results from the usage of a new unbinned method that uses spatial and energy information. At low energies, the limit obtained from this analysis almost reaches the high-energy tail of the FermiLAT sensitivity and giving the enhanced Galactic hadronic emission predicted by the reference models with respect to a conventional scenario, the obtained limits represent a strong constraint on a possible diffuse neutrino emission from the Galactic Plane.

Considering the flux upper limit with 90\% confidence level shown in Table 1 for the $50 \mathrm{PeV}$ cut-off, at most $18 \%$ of the cosmic neutrino events measured by IceCube with the HESE dataset can originate from CR diffusion. This corresponds to about 5.2 out of the 28.6 HESE with energy above $60 \mathrm{TeV}$ expected to be cosmic neutrinos, as reported in [22]. This limit is more restrictive than that allowed in $[10,20]$. The reference model produces a larger North/South asymmetry than the conventional scenario: more than $\sim 80 \%$ of the events are expected from the Southern hemisphere. Nevertheless, the contribution of the diffuse Galactic component to the difference between the observed number of HESE arising from the two hemispheres cannot be larger than 3.3 HESE, i.e. $\sim 10 \%$ of the full sky flux. As a result, the neutrino flux produced by the Galactic CR interaction with gas cannot explain by itself the IceCube spectral anomaly. These considerations are even more restrictive for the case of the $90 \%$ confidence level upper limit corresponding to a primary CR cut-off of $5 \mathrm{PeV}$, as evident from the predicted flux in Figure 2.

\section{References}

[1] M. Ackermann et al. Fermi-LAT Observations of the Diffuse $\gamma$-Ray Emission: Implications for Cosmic Rays and the Interstellar Medium. Astrophys. J., 750:3, 2012.

[2] D. Gaggero, D. Grasso, A. Marinelli, A. Urbano, and M. Valli. The Gamma-Ray and Neutrino Sky: A Consistent Picture of Fermi-LAT, Milagro, and IceCube Results. Astrophys. J. Lett., 815:L25, 2015.

[3] D. Gaggero, D. Grasso, A. Marinelli, M. Taoso, and A. Urbano. Diffuse cosmic rays shining in the Galactic center: A novel interpretation of H.E.S.S. and Fermi-LAT gamma-ray data.

arXiv:1702.01124, 2017. 
[4] D. Gaggero, D. Grasso, A. Marinelli, A. Urbano, and M. Valli. A Hadronic Scenario for the Galactic Ridge. PoS, ICRC2015:1126, 2015.

[5] KASCADE-Grande measurements of energy spectra for elemental groups of cosmic rays. Astropart. Phys., 47:54-66, 2013.

[6] M. G. Aartsen et al. First Observation of PeV-Energy Neutrinos with IceCube. Phys. Rev. Lett., 111(2):021103, 2013.

[7] M. G. Aartsen et al. The IceCube Neutrino Observatory - Contributions to ICRC 2015 Part II: Atmospheric and Astrophysical Diffuse Neutrino Searches of All Flavors. arXiv:1510.05223, 2015.

[8] M. G. Aartsen et al. Searches for Extended and Point-like Neutrino Sources with Four Years of IceCube Data. Astrophys. J., 796:109, 2014.

[9] M. G. Aartsen et al. Observation and Characterization of a Cosmic Muon Neutrino Flux from the Northern Hemisphere Using Six Years of IceCube Data. Astrophys. J., 833:3, 2016.

[10] A. Palladino, M. Spurio, and F. Vissani. On the IceCube spectral anomaly. J. Cosmol. Astropart. Phys., 12:045, 2016.

[11] A. Marinelli, D. Gaggero, D. Grasso, A. Urbano, and M. Valli. Interpretation of astrophysical neutrinos observed by IceCube experiment by setting Galactic and extra-Galactic spectral components. In EPJ Web of Conferences, volume 116, page 04009, 2016.

[12] M. Ageron et al. ANTARES: The first undersea neutrino telescope. Nucl. Instrum. Methods A, 656:11-38, 2011.

[13] Tino Michael. Neutrino point source search including cascade events with the ANTARES neutrino telescope. PoS, ICRC2015:1078, 2016.

[14] S. Adrián-Martínez et al. Searches for point-like and extended neutrino sources close to the galactic centre using the antares neutrino telescope. Astrophys. J., 786(1):L5, 2014.

[15] S. Adrián-Martínez et al. The first combined search for neutrino point-sources in the southern hemisphere with the antares and icecube neutrino telescopes. Astrophys. J., 823(1):65, 2016.

[16] S. Adrián-Martínez et al. Stacked search for time shifted high energy neutrinos from gamma ray bursts with the ANTARES neutrino telescope. Eur. Phys. J. C, 77(1), 2017.

[17] A. Albert et al. Time-dependent search for neutrino emission from x-ray binaries with the ANTARES telescope. arXiv:1609.07372, 2016.

[18] S. Adrián-Martínez et al. Constraints on the neutrino emission from the Galactic Ridge with the ANTARES telescope. Phys. Lett. B, 760:143-148, 2016.

[19] W. Atwood et al. Pass 8: toward the full realization of the Fermi-LAT scientific potential. arXiv:1303.3514, 2013.

[20] A. Neronov and D. Semikoz. Evidence the Galactic contribution to the IceCube astrophysical neutrino flux. Astropart. Phys., 75:60-63, 2016.

[21] S. Adrian-Martinez et al. Search for Cosmic Neutrino Point Sources with Four Year Data of the ANTARES Telescope. Astrophys. J., 760:53, 2012.

[22] M. G. Aartsen et al. Searches for Extended and Point-like Neutrino Sources with Four Years of IceCube Data Astrophys. J., 796:109, 2014 